\title{
Ingolf Dietrich
}

\section{Die Koka- und Kokainwirtschaft in Peru}

Vervuert Verlag, Frankfurt/Main, 1998, 314 S., DM 56,--

Weit über die Hälfte des zur Herstellung der weltweit meistkonsumierten illegalen Droge benötigten Rohstoffs Koka stammt aus Peru. Daran hat sich seit Beginn des Kokainbooms vor rd. 20 Jahren wenig geändert, auch wenn seit Mitte der 80er Jahre ein wachsender Teil der Ernte im Lande selbst zu Kokainbase und reinem Kokain (Kokainhydrochlorid) verarbeitet wird; zuvor war das aus den Blättern erzeugte Vorprodukt Kokapaste zur weiteren Verarbeitung größtenteils nach Kolumbien verbracht worden. Unbestritten ist, daß die volkswirtschaftliche Bedeutung des Drogensektors vor allem infolge der fallenden Kokapreise seit Ende der 80er Jahre zurückgegangen ist, wobei freilich einigermaßen verläßliche Angaben wegen der Klandestinität des illegalen Tuns nicht verfügbar sind. Wo wenig Konkretes bekannt ist, muß mit Schätzmethoden und indirekten Indikatoren gearbeitet werden; gleichzeitig begünstigt der kriminelle Charakter des Geschäfts vor allem in der Publizistik aber auch sensationsheischende Spekulationen. Daher werden zu diesem Problemkomplex höchst divergierende und partiell widersprüchliche Daten verbreitet. So weichen die Angaben über das Ausmaß der peruanischen Kokaproduktion im Verhältnis von 1:4 voneinander ab. Eine derart unbefriedigende Datenlage bietet kaum eine gute Voraussetzung zur Planung und Realisierung effizienter Gegenmaßnahmen. Deshalb ist verbesserten Kenntnissen über die Funktionslogik und/oder die quantitativen Merkmale des Drogengeschäfts gerade auch unter praxisorientierten Gesichtspunkten ein enormer Wert und Nutzen beizumessen.

Ingolf Dietrich geht es in seiner Studie um die Untersuchung der wirtschaftlichen und gesellschaftlichen Auswirkungen des peruanischen Koka-Kokain-Komplexes sowie um eine Darstellung und Bewertung der in diesem Bereich lancierten entwicklungspolitischen Gegenmaßnahmen. Nach einem skizzenhaften Überblick über die südamerikanische Kokainökonomie und einer kurzen landeskundlichen Einführung wendet sich der Autor in Kapitel 4 seinem eigentlichen Untersuchungsobjekt zu. Er beginnt mit grundlegenden Informationen zum Kokaanbau und beschreibt dann die Charakteristika des Ende der 70er Jahre im Huallaga-Tal einsetzenden Kokabooms. Interessant sind die Veränderungen, die es seither in der Produktion und im Vermarktungssystem des Rohstoffs und der Zwischenprodukte gegeben hat; darin spiegelt sich eine Anpassung an gewandelte Rahmenbedingungen, zu denen auch aus staatlichen Gegenmaßnahmen resultierende neue Risiken zählen.

Für eine Einschätzung der volkswirtschaftlichen Bedeutung des Koka(in)geschäfts bildet die zur Rohstofferzeugung genutzte Fläche einen fundamentalen Ausgangswert. Luftaufnahmen liefern aus diversen Gründen (Bewölkung, Anbau von Schattenpflanzen u.a.) wenig zuverlässige Erkenntnisse. Wegen der hohen Arbeitsintensität der Kokaproduktion stellt die "Verfügbarkeit von Arbeitskräften" einen brauchbaren indirekten Indikator zur Ermittlung der Anbaufläche dar. Die Ergebnisse einer Reihe von Untersuchungen stecken 
die quantitative Bandbreite der bislang vorgelegten Flächenschätzungen ab, wobei der daraus errechnete Mittelwert auch wegen der Nähe zu den eigenen Kalkulationen des Autors "als die wahrscheinlichste Größe betrachtet wird"(S. 76). Mehr als eine pragmatische Ausgangsbasis für die Ermittlung der Bedeutung des Drogensektors für Wirtschaft und Gesellschaft ist damit aber nicht gegeben. Dietrich nennt 14 Faktoren, die in diesem Zusammenhang relevant sind: vom durchschnittlichen Hektarertrag über die Produktionskosten auf jeder Verarbeitungsstufe bis zum Exportpreis und dem Anteil kolumbianischer Händler am Ausfuhrgeschäft. Auch wenn der Autor immer wieder auf die Schwäche der Daten und den dadurch bedingten tentativen Charakter der Resultate hinweist, wird an dieser Stelle die ganze Fragwürdigkeit seiner Bemühungen um eine Annäherung an die realen Dimensionen des Drogengeschäfts deutlich: Jede Schätzung bezüglich eines dieser Faktoren, so plausibel sie auch sein mag, hat zwangsläufig Auswirkungen auf das Rechenergebnis der nächsten Stufe. Die unsicheren Relationen zwischen 14 Faktoren erhöhen das Risiko einer kumulierten Fehlerwahrscheinlichkeit und vermögen den ermittelten Resultaten kaum die Aura einer gewissen Solidität zu verschaffen.

Unabhängig von diesem grundsätzlichen Einwand sind die im folgenden präsentierten Zahlen und Berechnungen nur zum Teil einleuchtend bzw. nachvollziehbar. So wird eine als besonders sorgfältig qualifizierte Untersuchung vorgestellt, aus der der Autor einige methodische Elemente für seine eigenen Kalkulationen übernimmt, deren quantitativen Angaben z.T. inkonsistent sind oder zumindest unrealistisch anmuten: So liegen die Kosten für die Herstellung von Kokapaste über dem entsprechenden Verkaufspreis des Zwischenproduktes, im Falle von Kokabase nur unwesentlich darunter. Ähnliche Mißverhältnisse tauchen auch in den eigenen Berechnungen des Autors auf, die er für die Jahre 1980-95 und für jeweils drei Flächenangaben (Maximal-, plausibler Mittel- u. konservativer Wert) erstellt. Diese Mängel relativieren das Erklärungspotential der Ergebnisse, können dies aber allein aufgrund des ohnehin unsicheren Terrains der Materie nicht gänzlich in Frage stellen. Dietrich veranschlagt den Wert der Kokablatterzeugung im erwähnten Zeitraum auf rd. $15 \%$ der landwirtschaftlichen Produktion. Die Deviseneinnahmen aus dem Drogenexport werden auf durchschnittlich 1 Mrd. US-\$ geschätzt, was je nach Jahr $20 \%$ bis $50 \%$ der legalen Ausfuhrerlöse gleichkam. Das Dollarangebot aus dem Drogengeschäft hat sich positiv auf die Stabilisierungspolitik Fujimoris, das Wirtschaftswachstum und die Zahlungsbilanz ausgewirkt. Der wichtigste direkte Effekt betrifft freilich die Arbeits- und Einkommensbasis, welche die Kokaproduktion und die Erzeugung von Kokapaste für über 150.000 Familien (ca. 750.000 Personen) geschaffen hat.

Die folgenden Ausführungen thematisieren den Drogenkonsum in Peru, die ökologischen Auswirkungen der Koka(in)produktion sowie die Implikationen des Drogengeschäfts für die Korruption auf verschiedenen Ebenen des Staatsapparats und wenden sich dann einem Vergleich der Wirtschaftlichkeit der Koka- u. Kokapasteproduktion mit anderen Agrarerzeugnissen zu. Während bei den Ausführungen zum Drogenkonsum einige Ungereimtheiten auffallen (die genannten Verbraucherzahlen sind offensichtlich zu hoch und mit den an anderen Stellen angegebenen nationalen Konsummengen unvereinbar), kommt der 
Autor hinsichtlich des letzten Aspekts trotz der von ihm selbst erwähnten Absatzprobleme alternativer Produkte zu dem Schluß, daß sich mit deren Anbau durchaus ein befriedigendes Einkommen erzielen lasse. Diese Einschätzung basiert auf den zu einem bestimmten Zeitpunkt ermittelten Preisen der alternativen Erzeugnisse, die freilich bei einer starken Ausweitung der Produktion nicht ohne weiteres das einmal erreichte Niveau halten können. Hinsichtlich der Darstellung der Rahmenbedingungen der Kokaerzeugung bleibt anzumerken, daß der über einen Zeitraum von mehreren Jahren wichtigen Rolle der Guerilla nur wenig Beachtung geschenkt wird, zudem wird deren Verhältnis zum Drogengeschäft unverständlicherweise als "strategische Allianz" charakterisiert.

Kapitel 5 beschäftigt sich mit der Politik zur Bekämpfung des Drogenhandels. Der Darstellung der internationalen Initiativen auf diesem Gebiet schließt sich eine kritische Bewertung der peruanischen Drogenpolitik an. Die Regierung Fujimori lehnt die von den USA präferierte vorrangig repressive Gegenstrategie ab und lancierte 1994 einen Plan, der eine schrittweise Reduzierung der Kokafläche mittels Bereitstellung wirtschaftlicher Alternativen für die Bauern vorsieht. Dietrich weist auf die hochgradige Ineffizienz der mit der Umsetzung der Programme betrauten staatlichen Stellen hin und betont den deklamatorischen Charakter der Ressortpolitik, der sich in der Wiederholung derselben oder ähnlicher Bestimmungen und Maßnahmen in immer neuen Gesetzen äußert, womit ein Engagement demonstriert werden soll, das mit der Realität wenig gemein hat. Die folgenden Abschnitte dieses Kapitels befassen sich mit der drogenpolitischen Strategie der USA sowie mit den entsprechenden Konzepten anderer Geberländer und der EU.

Kapitel 6, das den konkreten entwicklungspolitischen Maßnahmen zur Reduktion des Kokaanbaus gewidmet ist, bietet eine Fülle interessanter Informationen und Details. Beschrieben und bewertet werden die Programme der peruanischen Regierung, der UNOSonderorganisation UNDCP, der USA sowie ferner die Maßnahmen anderer ausländischer Geber. Während sich der Eigenbeitrag der peruanischen Regierung auf Infrastrukturmaßnahmen konzentriert, zielen die Projekte der UNDCP auf eine Substitution des Kokaanbaus durch andere Produkte, wobei neben der lokalen Weiterverarbeitung der Erzeugnisse auch die Sozialorganisation der Bauern gefördert wird. Weitere Projektelemente bestehen aus der Zurverfügungstellung von verbessertem Saatgut, der Ausschaltung des (zumeist parasitären) Zwischenhandels, Aus- und Fortbildungsmaßnahmen für die Bauern sowie der Einrichtung von Kreditfonds zur Finanzierung neuer Inputs. UNDCP ist in allen wichtigen Kokaanbauzonen des Landes aktiv. Im Einflußbereich der Projekte haben Tausende von Bauern die Kokaproduktion zugunsten anderer landwirtschaftlicher Erzeugnisse (u.a. Kakao, Tee, Palmherzen) aufgegeben, der Produktionswert legaler Kulturen hat sich zwischen 1985 und 1993 verdreifacht. Im Huallaga-Tal, dem größten Kokaanbaugebiet, hat sich die Zahl der Kooperativen infolge der Fördermaßnahmen der UNDCP von 1984 bis 1994 von zwei auf 150 erhöht. Auf der Negativseite der Projektbilanz im Huallaga und anderen Anbauzonen steht das Faktum, daß die Programme ohne ausländische Hilfe nicht weitergeführt werden können, daß gerade die ärmsten Bauern von den Maßnahmen nicht erfaßt 
wurden, die Anbauflächen an Steilhängen sich zur Substitution nicht eignen und bei der Projektplanung z.T. die Marktverhältnisse (Absatzchancen) zu wenig Beachtung fanden.

Die in den 80er Jahren von den USA in diversen Kokazonen finanzierten Programme hatten wenig positive Effekte und in einigen Gebieten zeitweise eher kontraproduktive Wirkungen, insofern als sie die Voraussetzungen für die Guerillatätigkeit verbesserten. Seit 1995 ist ein neues Programm in Kraft, das die alten Fehler vermeiden soll und im wesentlichen auf Substitution setzt. Auf der Haben-Seite der US-Programme finden sich Infrastrukturmaßnahmen und die Förderung der Gemeindeentwicklung. Drittwichtigster bilateraler Geber nach den USA und Kanada ist Deutschland, dessen Engagement sich stark auf drogenpolitische Entwicklungsprojekte (u.a. Förderung der Agrarforschung und -beratung) konzentriert.

Jenseits der inhärenten Schwächen und Defizite vieler Projekte gilt es zu betonen, daß der größte Teil der Kokaanbaugebiete und Kokabauern von den diversen Programmen gar nicht erfaßt wird. Wichtiger als die konzeptionelle Stimmigkeit der einzelnen Projekte erwies sich vielfach die Kokakonjunktur: Angesichts fallender Preise stieg die Bereitschaft zur Einstellung der Drogenrohstoffproduktion.

In seinen Schlußfolgerungen formuliert Dietrich die Voraussetzungen für eine erfolgreiche alternative Entwicklung in den Kokazonen. Dazu zählen auf internationaler Ebene ein Abbau der Agrarsubventionen der Industriestaaten, die negative Implikationen für viele Agrarprodukte der Andenländer haben und dadurch den Anbau von Koka begünstigen. Die bisher allzu begrenzten Mittel für Projekte alternativer Entwicklung könnten laut Dietrich durch Schuldentausch-Modelle ausgeweitet werden. Der Autor erwähnt zwar die große Bedeutung einer Senkung der Kokainnachfrage in den Industriestaaten, widmet diesem wichtigen Punkt aber lediglich eine halbe Seite. Die internen Voraussetzungen zur Verminderung der Kokaerzeugung durch entwicklungspolitische Maßnahmen bestehen auf der politischen Ebene aus einer Vielzahl von Faktoren - von Schritten zur Dezentralisierung und der Förderung der Partizipation über die Zurückdrängung der Korruption bis zur Herstellung von mehr Rechtssicherheit, wobei dem Autor durchaus bewußt ist, daß die meisten dieser Ziele sich allenfalls langfristig realisieren lassen. Auf der wirtschaftlichen Ebene wird eine Abkehr von einseitig neoliberalen Programmen und eine größere Kohärenz zwischen Wirtschaftspolitik und anderen Sektorpolitiken als unabdingbar angesehen. Auch und gerade in der Agrarpolitik sind Reformen erforderlich, um die Effizienz der drogenpolitischen Maßnahmen zu optimieren und Nachhaltigkeit zu erreichen. Unausgesprochen macht die riesige Kluft, die sich zwischen dem als notwendig Erachteten und den heutigen Realitäten auftut, überdeutlich, daß Peru seine herausragende Rolle im Koka/Kokaingeschäft auf absehbare Zeit nicht verlieren wird.

Von einigen Schwächen und kleinen Mängeln abgesehen, hat Dietrich mit seiner Untersuchung einen wertvollen Beitrag zum besseren Verständnis eines wichtigen Teilbereichs der internationalen Drogenproblematik geleistet.

Karl-Dieter Hoffmann 